\title{
Expression of Transcriptional Units Using Transmissible Gastroenteritis Coronavirus Derived Minigenomes and Full-length cDNA Clones
}

\author{
ISABEL SOLA, SARA ALONSO, CARLOS SANCHEZ, J. MANUEL \\ SANCHEZ-MORGADO, AND LUIS ENJUANES \\ Department of Molecular and Cell Biology, Centro Nacional de Biotecnología, CSIC. Campus \\ Universidad Autónoma. 28049 Madrid. Spain
}

\section{INTRODUCTION}

Many factors including RNA primary and secondary structure and protein-RNA interactions may regulate mRNA abundance. The nature of the transcription regulatory sequences (TRSs) and the extent of their complementarity to the leader $3^{\prime}$ end may be the most relevant and is discussed below. The TRSs include the core sequence (CS), previously named intergenic sequence (IG), that is a short conserved sequence element upstream the transcription units, and flanking sequences located upstream and downstream the CS. A helper dependent expression system based on transmissible gastroenteritis coronavirus (TGEV) derived minigenomes, encoding new subgenomic mRNAs, has been used to study the elements that regulate transcription in coronavirus. The optimization of TRSs can lead to the improvement of mRNA levels using both minigenomes and full-length cDNA clones. 


\section{OPTIMIZATION OF TRANSCRIPTION}

\subsection{Effect on mRNA levels of TGEV 5' and 3' core flanking sequences}

Using TGEV derived minigenomes including the heterologous gene $B$ glucuronidase (GUS) cloned under different TRSs, we have shown that the core CS sequence 5'-CUAAAC-3' is necessary and sufficient for mRNA expression. The absence of the CS (5'-CUAAAC-3') led to the complete abrogation of mRNA transcription. The insertion of the hexameric 5'CUAAAC-3' restored expression levels more than 400 -fold above the background. The addition of 5' upstream sequences flanking the core CS sequence from the TGEV $\mathrm{N}$ gene led to an increase in transcription up to 10 -fold. The expression in coronavirus can be driven by a TRS with $20 \mathrm{nt}$ in size, leading to $80 \%$ of the highest expression levels, produced by a TRSs of larger (88 nt) size (Alonso et al., 2000) (Figure 1).
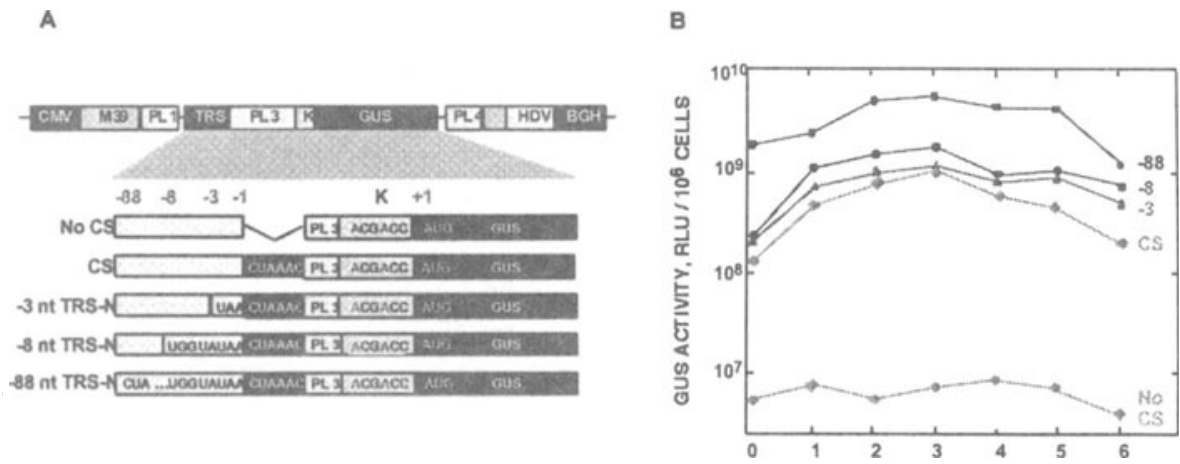

Figure 1. Effect of 5' flanking sequences on GUS expression. A. Expression cassettes including the GUS gene under different TRSs. TRSs include the core sequence 5'-CUAAAC3' and 5' flanking sequences derived from TGEV $\mathrm{N}$ gene with different lengths. The minigenome M39 is cloned behind the cytomegalovirus promoter (CMV). B. GUS activity produced by constructs shown in A during six passages in cell culture. PL, polylinker. K, Kozak type sequence. An, poly A. HDV, hepatitis delta-virus ribozyme. BGH, bovine growth hormone termination and polyadenilation signals. RLU, relative luminometric units.

The sequences downstream the core CS of seven viral genes (S, 3a, 3b, $\mathrm{E}, \mathrm{M}, \mathrm{N}$, and 7) have sizes ranging from 3 to $37 \mathrm{nt}$. Expression modules in which the 5' flanking sequence was kept constant, and the 3' CS flanking sequences were provided by each of the seven viral genes, led to similar expression levels of the heterologous mRNA, with the exception of that 
from ORF 3a. This construct gave expression levels 5- to 10-fold lower. Thus, there was no correlation between the length of the 3 ' flanking sequences and the expression level. Two non viral 3' flanking sequences were analyzed for mRNA expression. The sequence including an optimized Kozak motif produced mRNA levels as high as the best viral derived sequence. In contrast, when the sequences flanking the 3' end of the CS were engineered extending with $12 \mathrm{nt}$ their potential basepairing with the 3 ' of the leader, mRNA levels were abrogated (Alonso et al., 2000). The optimization of TRSs led to the expression of high amounts of heterologous protein ( 1 to $8 \mu \mathrm{g} / 10^{6}$ cells), maintained for around 10 passages. These expression levels should be sufficient to elicite protective immune responses.

\subsection{Influence of the insertion site}

Using a TGEV derived helper dependent expression system, the expression cassette containing the GUS gene was inserted at different nucleotide distances from the $5^{\prime}$ end. The expression levels increased from the 5' to 3' end by one thousand-fold (Alonso et al., 2000). The insertion site close to the $5^{\prime}$ end probably has affected essential primary or secondary structures required for minigenome replication, thus reducing the significance of this result in relationship to the insertion site. Furthermore, no difference in expression levels was observed with the MHV system, when the flanking sequences were kept constant for all insertion sites, suggesting that the location of the insertion site per se may not affect transcription levels and that the differences observed with TGEV were mostly due to the CS-flanking sequences.

\subsection{Stability of TGEV helper dependent expresssion systems}

GUS expression using TGEV derived minigenomes was observed for about ten passages (Alonso et al., 2000). In general, the insertion of a heterologous gene such as GUS into TGEV derived minigenomes led to a 50 -fold reduction in the levels of the minigenome RNA replication. The limited stability of the helper dependent expression systems is most likely due to the foreign gene, since TGEV minigenomes, in the absence of the heterologous gene, are efficiently rescued for at least 30 passages (Izeta et al., 1999). The recombination frequency in MHV, TGEV, and IBV may be inversely proportional to the stability of the recombinants expressing a foreign gene. 


\section{SINGLE GENOME TGEV VECTORS}

To overcome the limited stability associated to RNA minigenomes, single genome TGEV vectors have been developed either by targeted recombination or by engineering an infectious cDNA encoding TGEV.

\subsection{Vectors constructed by targeted recombination}

Targeted recombination mediated by two cross-overs allowed the replacement of the S gene of a respiratory strain of TGEV by the S gene of enteric TGEV, leading to the isolation of viruses with a modified tropism and virulence (Sánchez et al., 1999). A new strategy for the selection of TGEV recombinants was based in the elimination of the parental replicative virus by its simultaneous neutralization with two mAbs. The frequencies of the targeted recombination event in recombination for MHV and TGEV were found higher than the standard prediction for the recombination frequency of a multiple cross-over, suggesting that the alignment of two templates is the rate-limiting event in this recombination.

\subsection{Coronavirus vectors derived from an infectious cDNA clone}

Using the TGEV infectious cDNA (Almazan et al., 2000), the green fluorescent protein (GFP) gene was cloned by replacing the non-essential 3a and $3 \mathrm{~b}$ genes, leading to an engineered genome with high stability and expression levels around $40 \mu \mathrm{g} / 10^{6}$ cells (Figure 2) (Sola et al., 2000).

A

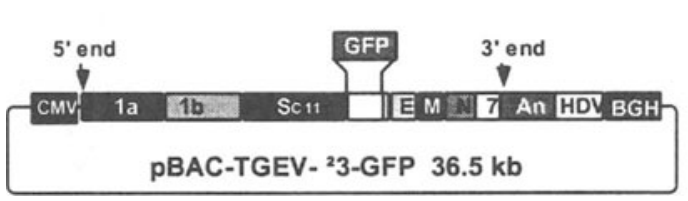

B

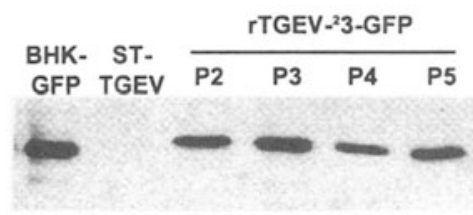

Figure 2. Expression of GFP using an infectious TGEV cDNA clone. A. Genes 3a and $3 b$ were deleted in the TGEV infectious cDNA cloned in a bacterial artificial chromosome (BAC). GFP $(0.72 \mathrm{~kb})$ was inserted within the position of the deleted genes after the TRS of gene $3 \mathrm{a}$, leading to a replication competent cDNA, rTGEV- $\triangle 3-G F P$. B. Western-blot analysis of GFP expression in cell culture along passages P2, P3, P4, P5. CMV, immediate-early cytomegalovirus promoter. SC11, S gene of PUR-C11 TGEV strain. An, poly A. HDV, hepatitis delta-virus ribozyme. $\mathrm{BGH}$, bovine growth hormone termination and polyadenylation signals. BHK-GFP, positive control of BHK cells expressing GFP. STTGEV, negative control of ST cells infected with $w t$ TGEV. 


\section{CONCLUSION}

Both helper-dependent expression systems, based on two components, and single genomes constructed by targeted recombination or by using an infectious cDNA have been developed. The sequences that regulate transcription have been characterized. Single genome coronavirus vectors have been constructed efficiently expressing a foreign gene such as GFP. Thus, a new avenue with high potential has been opened for coronaviruses which have unique properties, such as a long genome size and enteric tropism, that makes them of high interest as expression vectors for vaccine development and gene therapy. The possibility of engineering the tissue and species tropism will make coronavirus very flexible expression systems, since the same vector could be modified to target expression to different organs and animal species, including humans.

\section{ACKNOWLEDGMENTS}

The work was supported by grants from the Comisión Interministerial de Ciencia y Tecnología (CICYT), la Consejería de Educación y Cultura de la Comunidad de Madrid, and the European Community (Key Action 2: Infectious Diseases).

\section{REFERENCES}

Almazan, F., González, J. M., Pénzes, Z., Izeta, A., Calvo, E., Plana-Durán, J., and Enjuanes, L. (2000). Engineering the largest RNA virus genome as an infectious bacterial artificial chromosome. Proc. Natl. Acad. Sci. USA 97, 5516-5521.

Alonso, S., Izeta, A., Sola, I., and Enjuanes, L. (2000). Transcription regulatory sequences in transmissible gastroenteritis coronavirus. Submitted.

Izeta, A., Smerdou, C., Alonso, S., Penzes, Z., Méndez, A., Plana-Durán, J., and Enjuanes, L. (1999). Replication and packaging of transmissible gastroenteritis coronavirus-derived synthetic minigenomes. J. Virol. 73, 1535-1545.

Sánchez, C. M., Izeta, A., Sánchez-Morgado, J. M., Alonso, S., Sola, I., Balasch, M., PlanaDurán, J., and Enjuanes, L. (1999). Targeted recombination demonstrates that the spike gene of transmissible gastroenteritis coronavirus is a determinant of its enteric tropism and virulence. J. Virol. 73, 7607-7618.

Sola, I., Alonso, S., Plana-Durán, J., and Enjuanes, L. (2000). Heterologous gene expression with a single genome derived from transmissible gastroenteritis coronavirus. J. Virol., Submitted for publication. 